Diabetologia 10, 775-782 (1974)

(C) by Springer-Verlag 1974

\title{
Insulin Secretory Response of Diabetics during the Period of Improvement of Glucose Tolerance to Normal Range
}

\author{
K. Kosaka ${ }^{1}$, R. Hagura ${ }^{2}$, T. Kuzuya ${ }^{3}$, and N. Kuzuya ${ }^{2}$ \\ 1 Third Dept. of Internal Medicine, Faculty of Medicine, University of Tokyo, Hongo, Tokyo, ${ }^{2}$ The Institute for \\ Adult Diseases, Asahi Life Foundation Nishishinjuku, Tokyo, ${ }^{3}$ Dept. of Medicine, Jichi Medical School, Minamikochi- \\ machi, Tochigi-ken, Japan \\ Received: January 3, 1974, and in revised form: August 5, 1974
}

\begin{abstract}
Summary. Serum insulin responses to $100 \mathrm{~g}$ oral glucose, intravenous tolbutamide, and oral glucose plus intravonous glucagon and tolbutamide, were studied in patients who were definitely diabetic but subsequently improved to have normal glucose tolerance following treatment. "Definite diabetes" was diagnosed when the pationt had had fasting blood sugar higher than $150 \mathrm{mg} /$ $100 \mathrm{ml}$ or had clear diabetic retinopathy plus glucose intolerance. This improved group, whether nonobese or obese, had significantly decreased insulin responses during glucose tolerance test and glucose-glucagon-tolbutamide test, but the insulin response to intravenous tolbutamide was not significantly different from the control. In contrast, in the secondary diabetes group, whose glucose
\end{abstract}

intolerance might be attributable to other diseases than diabetes, insulin response to glucose was enhanced, and was normalized when glucose tolerance became normal. The insulin response to glucose of the prediabetes group (i.e. with both parents diabetic) with normal glucose tolerance was intermediate between those of the healthy and diabetes groups. It seems that the low insulin response to glucose is a less easily corrigible feature than glucose intolerance and probably constitutes one of the most fundamental abnormalities in primary diabetes.

Key words: Insulin response, oral GTT, tolbutamide test, glucose-glucagon-tolbutamide test, definite diabetes, prediabetes, secondary diabetes, retinopathy.
A number of previous studies have demonstrated that insulin secretory responses to glucose or other stimuli are generally diminished in moderately or severely diabetic patients. In cases of mild diabetes or so-called chemical diabetes, although plasma insulin often reaches a higher than normal level following a glucose load, the response is, in general, delayed and if the influence of obesity [1] and the increment of blood sugar $[2,3]$ are taken into consideration, it is also regarded as subnormal. Some contradictory results have also been reported, however [4].

Decreased insulin response to glucose is considered to be characteristic by some investigators not only for diabetic patients but also for prediabetic subjects who have a hereditary disposition to diabetes $[5,6]$. Cerasi and Luft [6] proposed a hypothesis that a low insulin response to glucose infusion could serve as a genetic marker of diabetes, and that true diabetes may develop only in "low insulin-responders".

The insulin response of a diabetic patient can vary considerably with the change of metabolic state [7]. The aim of the present study was to investigate the insulin response of diabetic patients during the period of best improvement of their diabetic state.

It is rather uncommon for a definitely diabetic patient to improve to completely normal glucose tolerance. We have had the opportunity to study the insulin response of 23 such cases and found that their insulin responses remained significantly lower than normal despite normal glucose tolerance at the time of examination.

\section{Materials and Methods}

The principal subjects of this study were the patients with definite diabetes, and subsequently with normal glucose tolerance. Among about 2000 patients attending the diabetes outpatient clinics of Tokyo Women's Medical College and of University of Tokyo Hospitals, 23 such patients were found. They are called "improved group". Among them, 3 were initially treated with insulin, 6 with sulfonylureas, and 14 only on diet. None of them however, needed any drugs at the time of examination. During improvement, 8 patients lost weight by $5 \mathrm{~kg}$ or more. In the present paper, definite diabetes is defined when we have confirmed that the patient has had a fasting blood sugar (FBS) higher than $150 \mathrm{mg} / 100 \mathrm{ml}$ during the period of observation, or when the patient has clear diabetic retinopathy with microaneurysms plus diabetic glucose tolerance.

This "improved" group was compared with the following groups for their insulin responses. 1. Control group: 75 subjects (50 non-obese and 25 obese) who had normal glucose tolerance and were without a family history of diabetes, and without any acute or chronic metabolic diseases.

2. Diabetes groups: 139 cases with definite diabetes, who had either FBS higher than $150 \mathrm{mg} / 100 \mathrm{ml}$ initially or diabetic retinopathy, but with unspecified FBS at the time of GTT. They were either untreated, or, if treated on oral antidiabetic drugs, drugs were withheld at least for a week before the GTT. 3. Predia- 
betes group: 16 subjects, both parents diabetic, who had normal glucose tolerance. 4. Secondary diabetes group : 2 cases on corticosteroid treatment for systemic lupus erythematosus or hepatitis, 3 cases of hyperthyroidism and 3 cases of acute hepatitis, who all had diabetic glucose tolerance, but who eventually came to have normal glucose tolerance.

All the tests were carried out after an overnight fast. The glucose tolerance test was performed using $300 \mathrm{ml}$ Trelan G 50® (Simizu Seiyaku Co. ${ }^{1}$. It has been reported that plasma glucose and insulin responses following administration of this solution are essentially the same as those after a standard $100 \mathrm{~g}$ glucose load [8-10]. Blood sugar determination was made with blood samples taken from a cut on the ear lobe before and $30,60,90,120$ and $180 \mathrm{~min}$ after the ingestion of the test solution. Samples for serum insulin assay were withdrawn at the same time from an arm vein into glass tubes. Blood sugar was measured by HagedornJensen's method [11], which gave values $10-15 \mathrm{mg} /$ $100 \mathrm{ml}$ higher than the glucose-oxidase method in our laboratory. Serum insulin was assayed by a doubleantibody technique [12].

The criteria for the evaluation of glucose tolerance curve were based on the recommendation of the Japan Diabetic Society [13]; the glucose tolerance curve in which $60 \mathrm{~min}$ and $120 \mathrm{~min}$ values were higher than 200 and $180 \mathrm{mg} / 100 \mathrm{ml}$, respectively, was defined as diabetic, and the glucose tolerance curve in which $60 \mathrm{~min}$ and $120 \mathrm{~min}$ values were less than 180 and $140 \mathrm{mg} / 100 \mathrm{ml}$ respectively, was defined as normal. Intermediate curves were defined as borderline.

The intravenous tolbutamide test was carried out by injecting $0.5 \mathrm{~g}$ tolbutamide intravenously in $1 \mathrm{~min}$. Blood samples were collected before and 1, 5, 10, 20, $30,40,50,60$ and $90 \mathrm{~min}$ after the injection.

Glucose-glucagon-tolbutamide test was performed according to the method of Ryan and others [14]. After a fasting blood sample was withdrawn, $300 \mathrm{ml}$ of Trelan $\mathrm{G}^{\circledR}$ was given orally. Another blood sample was taken at $30 \mathrm{~min}$ and then $1 \mathrm{mg}$ glucagon and $0.5 \mathrm{~g}$ tolbutamide were quickly injected intravenously. Blood samples were taken 1, 5, 10, 30, 60 and $120 \mathrm{~min}$ after the injection.

\section{Results}

\section{Insulin Responses During GTT}

Insulin responses following an oral glucose load (mean $\pm \mathrm{SD}$ ) of control, prediabetes, improved groups and diabetic groups are shown in Fig. 1. Because of the well-known effect of obesity on serum insulin, non-obese subjects (less than 110\% ideal

$1300 \mathrm{ml}$ Trelan $\mathrm{G} \circledast$ contains carbonated and flavoured hydrolysates of carbohydrate corresponding to $100 \mathrm{~g}$ glucose. The composition of sugars are as follows: glucose $35.5 \%$, maltose $19.5 \%$, maltotriose $12.5 \%$ and higher saccharides $32.5 \%$. standard body weight ${ }^{2}$ ) and obese subjects (more than $110 \%$ standard body weight) are shown separately. Although the glucose tolerance curves of the first three groups all fell within the normal range, the improved group had slightly higher mean blood sugar values than the control group after oral glucose load. The diabetic groups were subdivided according to the FBS at the time of GTT.

Serum insulin responses in the control, prediabetes and improved groups were markedly different despite similar, normal glucose tolerances. In the control group, mean levels of serum insulin reached a peak of $86 \mu \mathrm{U} / \mathrm{ml}$ (non-obese) or $127 \mu \mathrm{U} / \mathrm{ml}$ (obese) at $30 \mathrm{~min}$. The insulin response was markedly depressed in the improved group. The insulin response of the prediabetes group was intermediate. In the diabetes groups, the insulin responses were generally low and progressively decreased with increasing FBS. When the FBS levels were lower than $160 \mathrm{mg} / 100 \mathrm{ml}$, the peak insulin levels were not necessarily low, but the $30 \mathrm{~min}$ values were clearly subnormal. In most groups, obese subjects had higher insulin levels than non-obese subjects in the same group. The individual data of blood sugar and serum insulin concentrations of the improved group are presented in Table 1. Cases No. 1-10 and No. 16-22 were diagnosed as definite diabetes because their initial FBS were higher than $150 \mathrm{mg} / 100 \mathrm{ml}$, and cases No. 11-15 and No. 23 had diabetic retinopathy. With a few exceptions, their insulin values $30-120$ min after the glucose load were less than the range of corresponding values in the control group. This was true both for non-obese and obese cases.

The decrease in insulin response in the improved group is more clearly demonstrated when the ratios $(\Delta \mathrm{IRI} / \Delta \mathrm{BS})$ of the increments of insulin $(\mu \mathrm{U} / \mathrm{ml})$ to blood sugar $(\mathrm{mg} / 100 \mathrm{ml}) 30 \mathrm{~min}$ after glucose load were calculated (Fig. 2). The $\Delta I R I / \Delta B S$ values of the improved group and the diabetic groups were markedly lower and those of the prediabetes group were moderately lower than the control values. As an index of overall insulin response, the sums of 6 insulin values ( $\Sigma$ IRI) obtained during GTT are shown in Fig. 3 together with the sum of 6 blood sugar values ( $\Sigma B S$ ). The general pattern is similar to that of Fig. 2, in that the insulin response was decreased moderately in the prediabetes group and markedly in the improved group. In the diabetes groups, $\Sigma$ IRI was moderately or markedly decreased.

In the improved group, cases were examined twice for their insulin response, before and after normalization of their GTT. As shown in Fig. 4, there was a poor response of serum insulin in both tests. The insulin levels after improvement were not significantly different from those before. Fig. 4 also shows the data of "secondary diabetics", who had had a diabetic glucose tolerance initially, possibly due to causes other than

2 Ideal body weight was calculated from the body height according to the following equation (15). Ideal body weight $(\mathrm{Kg})=[$ body height $(\mathrm{cm})-100] \times 0.9$. 
primary diabetes, and later had normal glucose tolerance. In this secondary diabetes group, the insulin response was markedly higher than the control when the glucose tolerance was diabetic, but decreased to the normal range after the normalization of glucose tolerance. The values of $\Delta \mathrm{IRI} / \Delta \mathrm{BS}$ remained within the normal range in the secondary diabetes group whether the results of GTT were diabetic or normal.

\section{Insulin Responses to Intravenous Administration of $0.5 \mathrm{~g}$ Tolbutamide}

As shown in Table 2, serum insulin began to increase within one minute and reached the maximum 5 min after the injection of tolbutamide, except for the diabetic group with FBS higher than $160 \mathrm{mg} / 100 \mathrm{ml}$, which had a more sluggish insulin response. In the improved group, the peak insulin level was somewhat lower, but was not significantly different from that in the healthy control group. The peak insulin level was lower in the diabetes groups, and especially when FBS was higher than $160 \mathrm{mg} / 100 \mathrm{ml}$.

The fall of blood sugar was most prompt and marked in the control group, and tended to be delayed and less marked in other groups.

\section{Insulin Responses to the Glucose-Glucagon-Tolbutamide Test}

In the control group, serum insulin increased promptly to a peak of $605 \pm 343 \mu \mathrm{U} / \mathrm{ml} 5 \mathrm{~min}$ after intravenous injection of glucagon and tolbutamide

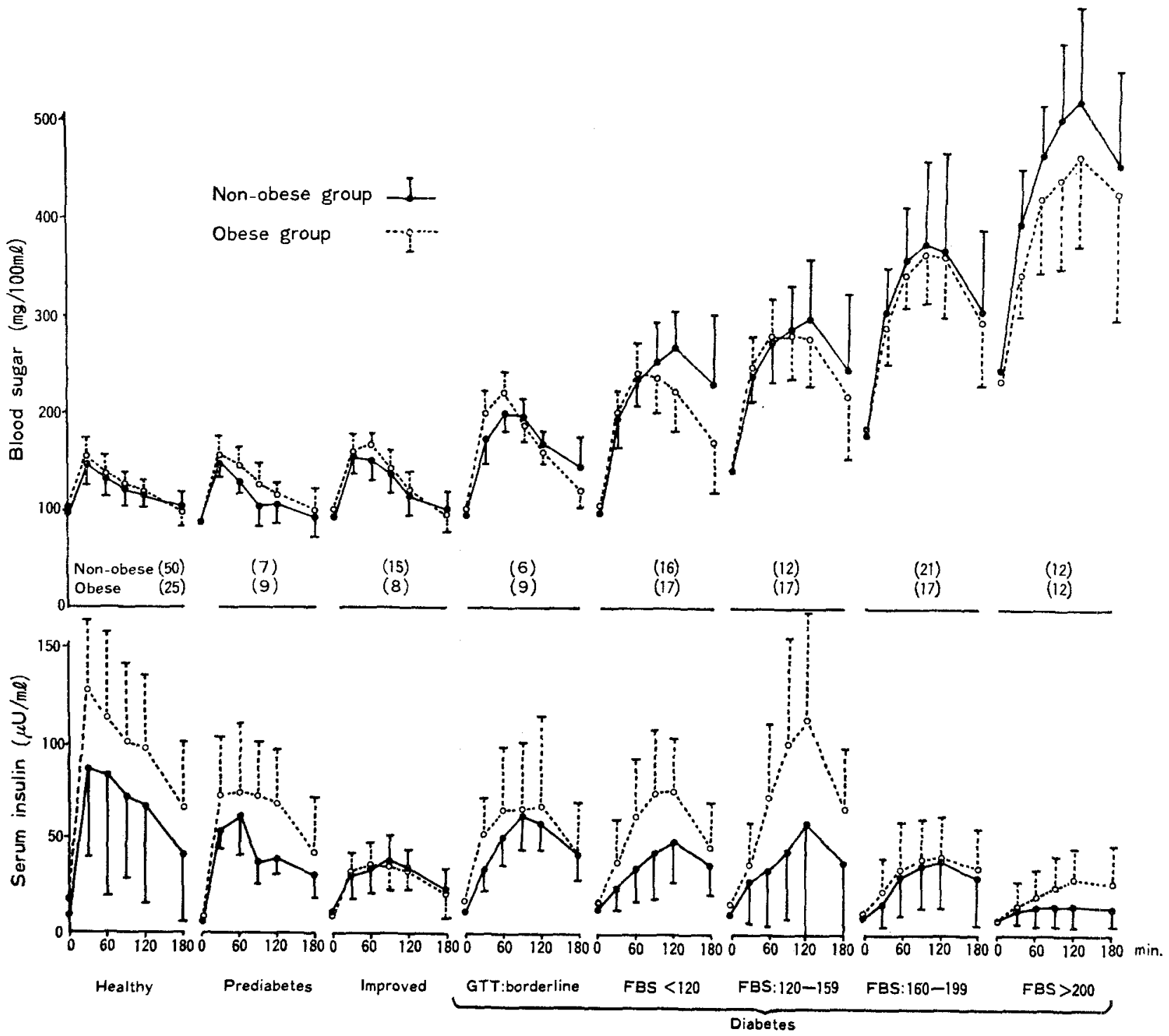

Fig. 1. Changes in blood sugar and serum insulin concentrations (mean \pm SD) during $100 \mathrm{~g}$ GTT in the healthy control, prediabetes, improved and diabetes groups. The number of non-obese or obese subjects in each group is shown in parentheses 
K. Kosaka et al.: Insulin Response during Improvement of Diabetes

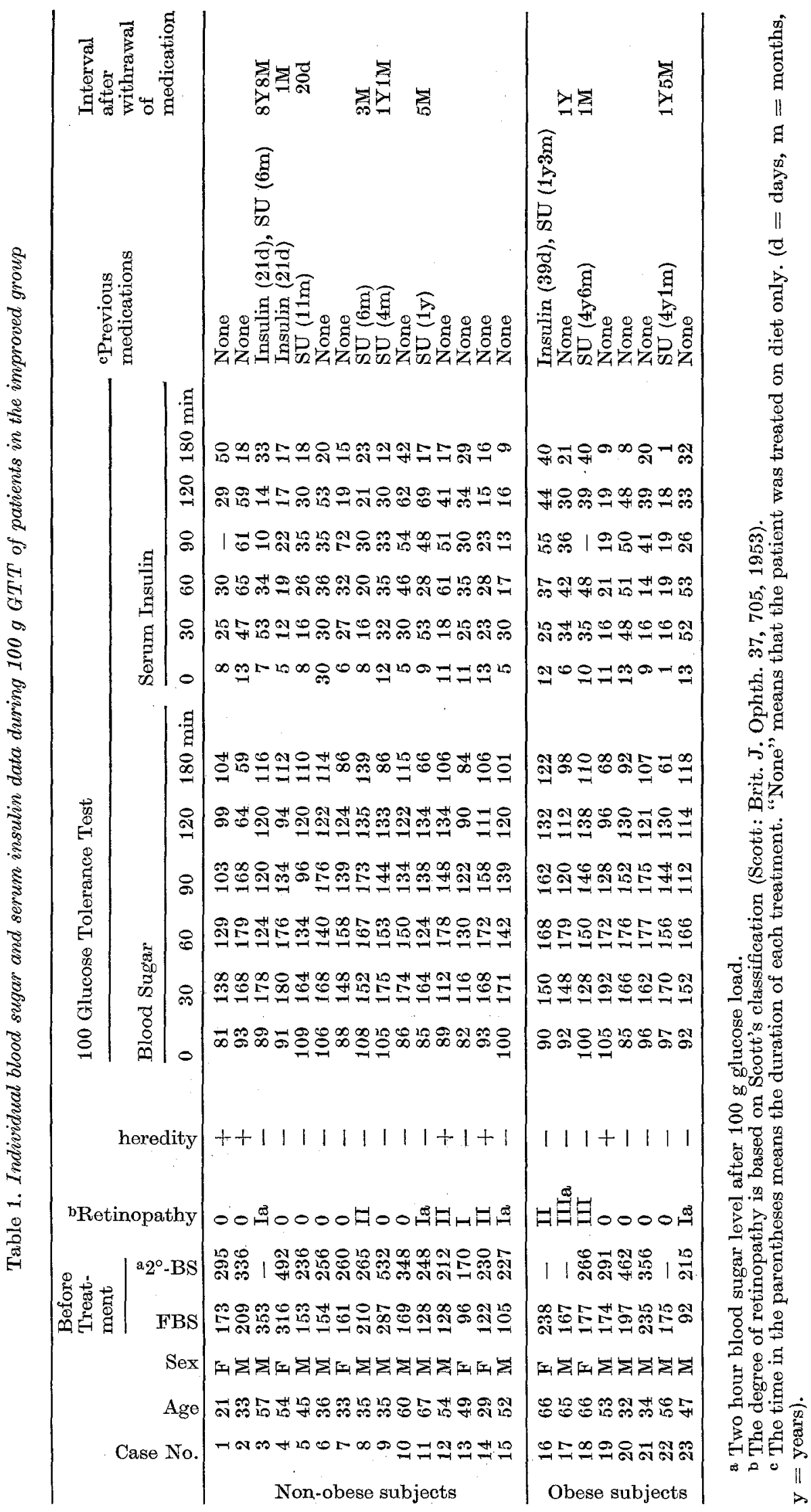



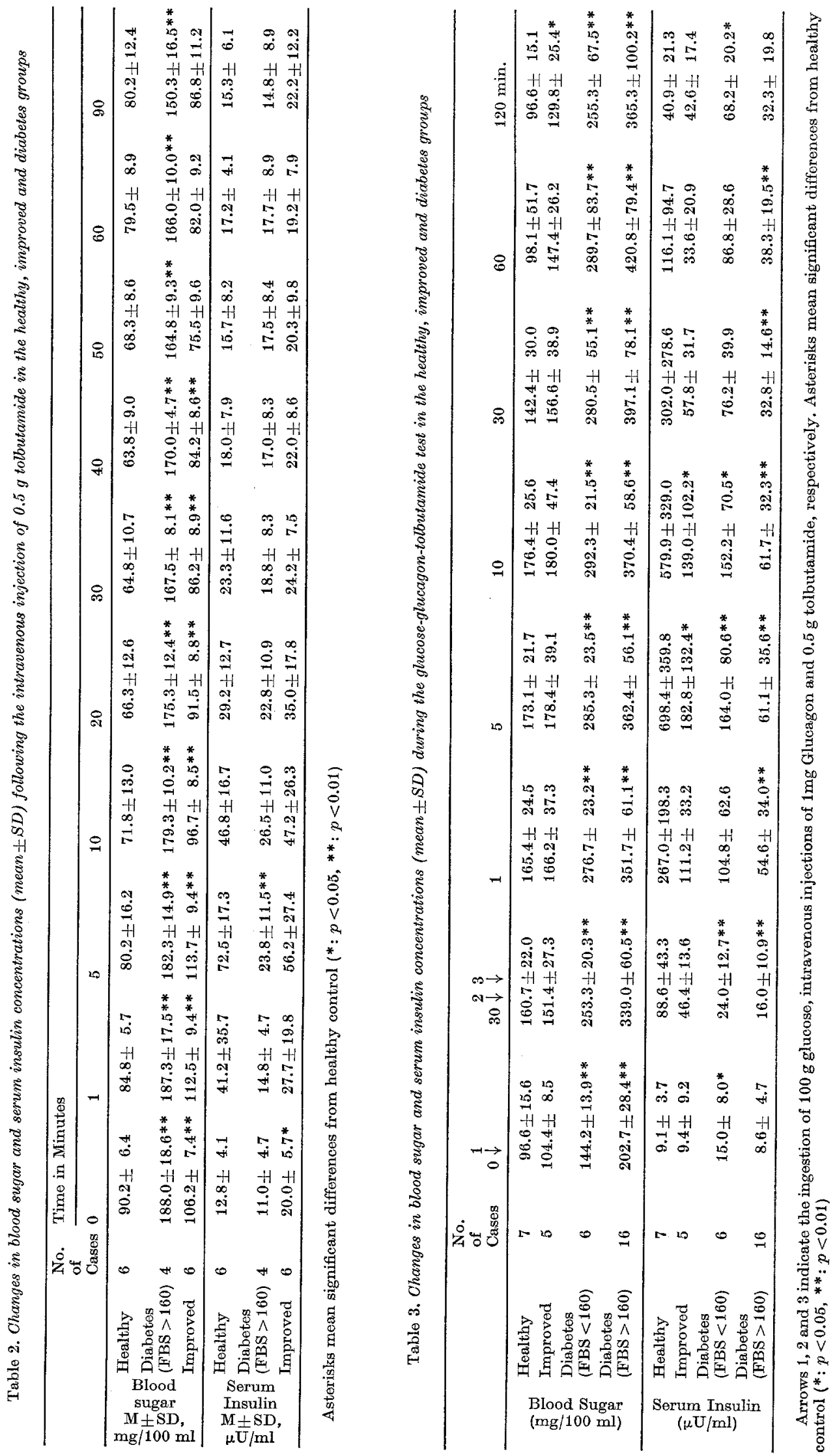
(Table 3). The insulin responses in the improved group and the diabetic groups were both much smaller than control.

\section{Discussion}

Although diabetes mellitus is often diagnosed on the basis of glucose intolerance, it is obvious that simple glucose intolerance is inadequate for the diag. nosis of true primary diabetes, in view of the diversity of the factors which can impair glucose tolerance. As our attention was particularly focussed upon the insulin response after normalization of glucose tolerance, chemical diabetes and without retinopathy, were excluded from our study.

In conformity with most previous reports, definitely diabetic patients thus selected were all low insulin responders to oral glucose, if their blood sugar values were taken into consideration to evaluate insulin responses. Insulin responses were markedly depressed in patients who had definite diabetes, but with normal glucose tolerance at the time of GTT. Luft and associates [16] reported that the low insulin response of diabetics with acromegaly remained low after im. provement of glucose tolerance by hypophysectomy. Our results seem to indicate that the persistence of a

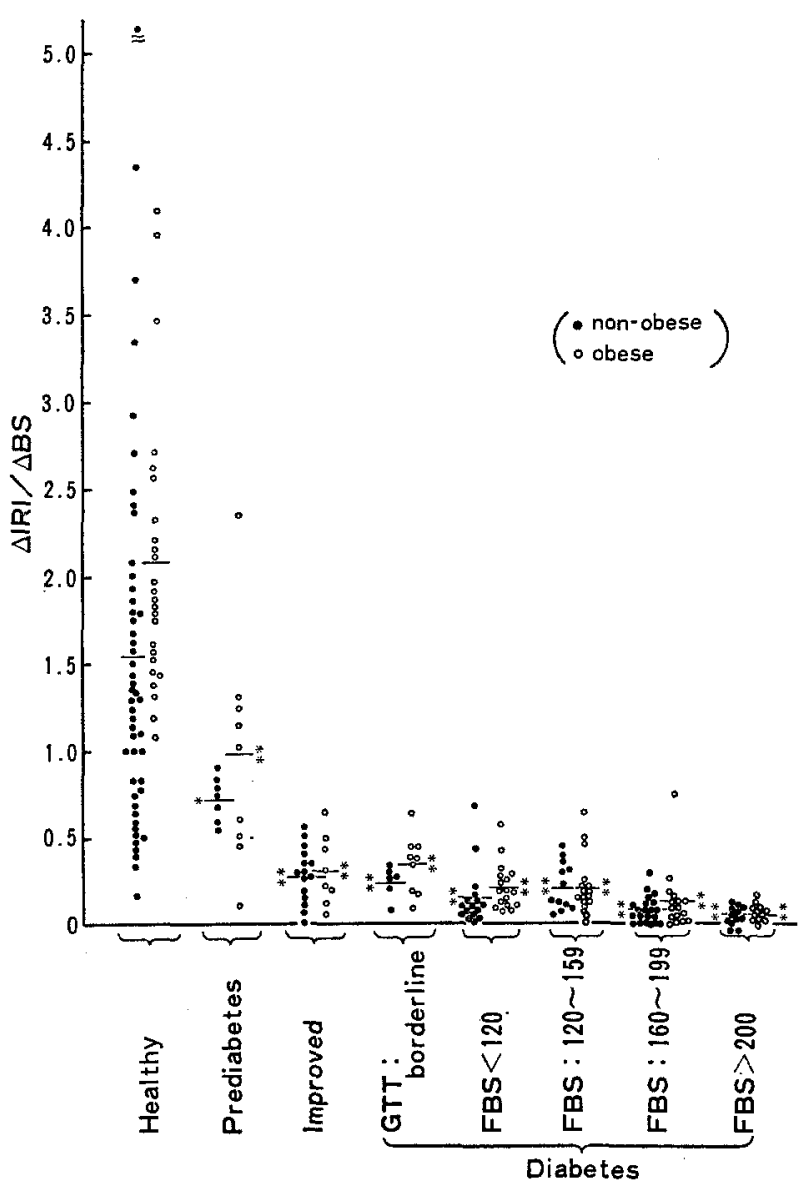

Fig. 2. The ratios $(\Delta \mathrm{IRI} / \Delta \mathrm{BS})$ of the increment of insulin $(\mu \mathrm{U} / \mathrm{ml})$ to the increment of blood sugar $(\mathrm{mg} / 100 \mathrm{ml}) 30$ min after $100 \mathrm{~g}$ glucose load in the healthy control, prediabetes, improved and diabetes groups. Asterisks mean the significance of difference from corresponding controls (* $P<0.05, * * P<0.01$ )

we attempted to select patients who had unequivocal or definite diabetes by either of two criteria. Our criteria of FBS higher than $150 \mathrm{mg} / 100 \mathrm{ml}$ before treatment, or the presence of definite diabetic retinopathy plus impaired GTT are rather arbitrary. Cases who might have true primary diabetes, but at the stage of

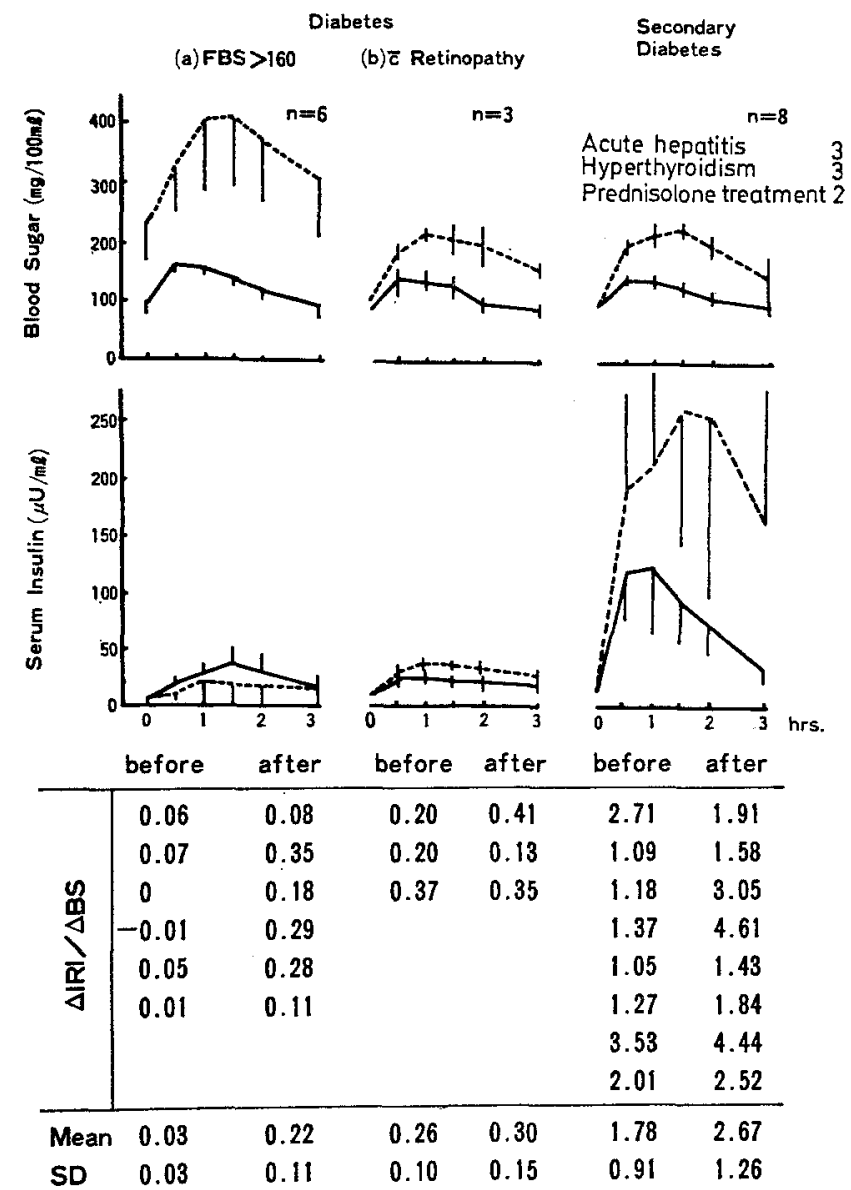

Fig. 3. Changes in blood sugar, serum insulin concentrations (mean $\pm \mathrm{SD}$ ) and $\Delta \mathrm{IRI} / \Delta \mathrm{BS}(30 \mathrm{~min})$ during $100 \mathrm{~g}$ GTT in the improved and secondary diabetes groups before and after normalization of their glucose tolerance

low insulin response after improvement of GTT is a general phenomenon in diabetics in whom there is no specific diabetogenic factor. The prediabetes group also had lower insulin responses than the control, in keeping with the data of previous reports $[5,6]$. The decrease in insulin response in prediabetes, however, was less marked than that of the improved group. 
These data suggest that the impaired insulin release by glucose is a less easily corrigible defect in primary diabetes than is glucose intolerance. It probably constitutes one of the most inherent and fundamental abnormalities in the pathophysiology of diabetes during the phases of prediabetes and of improvement.

In contrast, in the secondary diabetes patients whose glucose intolerance might be attributable to other diseases than diabetes, insulin responses were enhanced when the GTT was diabetic and normalized when GTT became normal. As $\Delta$ IRI/ $\Delta$ BS ratios were within the normal range in these patients, it may be that the B-cells responded normally to the changes in blood sugar during the course of their primary diseases.

It has been suggested that impairment of insulin response in prediabetes or mild diabetes is a selective defect to glucose stimulation. Insulin responses in mild diabetics may be nearly normal after tolbutamide [17] or isoproterenol [18], but severely impaired following intravenous glucose. Some investigators $[5,19]$ reported a subnormal insulin release after tolbutamide in prediabetic subjects. However, our data showed that tolbutamide induced smaller insulin responses in the improved and diabetes groups than normal, but the decreases in insulin responses were not so marked after tolbutamide as those following glucose stimulation. Selective impairment of insulin release to glucose may exist to some extent in these cases. The marked decrease in insulin response to the glucose-glucagontolbutamide test in the improved and diabetes groups may be explained by their insensitivity to glucose stimulation.

Several questions emerge from the subnormal insulin response to glucose in the improved and prediabetes groups. Normal glucose tolerance despite low insulin release might mean some compensatory adaptation in the body. It is not known whether low insulin response means lack of insulin in the body. It is possible that glucose tolerance is a too insensitive index to be affected by a very minor lack of insulin. The possibility exists that such a small deficiency of insulin might be relevant to diabetic microangiopathy, which may appear even in the stage of prediabetes [20] and progresses even with the best means of treatment of diabetes. Another question is whether the decreased insulin release in prediabetes exists from birth or whether it appears and progresses during growth. That the prediabetes group had intermediate insulin responses between the control and diabetes group might support the latter view or simply indicate that not all the subjects with both parents diabetic have a genetic disposition to diabetes. A long-term follow up study of the same prediabetic cases is necessary to clarify this problem.

\section{References}

1. Bagdade, J.D., Bierman, E.L., Porte, D. Jr.: The significance of basal insulin levels in the evaluation of the insulin response to glucose in diabetic and nondiabetic subjects. J. clin. Invest. 46, 1549-1557 (1967)

2. Seltzer, H.S., Allen, E. W., Herron, A.L. Jr., Brennan, M.T.: Insulin secretion in response to glycemic stimulus: Relation of delayed initial release to carbohydrate intolerance in mild diabetes mellitus. J. clin. Invest. 46, 323-335 (1967)

3. Perley, M.J., Kipnis, D.M. : Plasma insulin responses to oral and intravenous glucose - Studies in normal and diabetic subjects. J. clin. Invest. 46, 1954-1962 (1967)

4. Reaven, G.M., Shen, S.W., Silvers, A., Farquhar, J.W.: Is there a delay in the plasma insulin response of patients with chemical diabetes mellitus ? Diabetes 20, 416-423 (1971)

5. Boden, G., Soeldner, J.S., Gleason, R.E., Marble, A.: Elevated serum human growth hormone and decreased serum insulin in prediabetic males after intravenous tolbutamide and glucose. J. clin. Invest. 47, $729-739$ (1968)

6. Cerasi, E., Luft, R.: "What is inherited - what is added" hypothesis for the pathogenesis of diabetes mellitus. Diabetes 16, 615-627 (1967)

7. Kosaka, K., Hagura, R., Saito, R., Tsukamoto, F., Kuzuya, T.: Changes in plasma insulin and glucose tolerance in stable diabetes in a young woman. Diabetes 18, 487-492 (1969)

8. Tkeda, Y., Saito, H., Anzawa, R., Sano, T., Yaginuma, N., Shimizu, N.: A new carbohydrate solution, SDT-25 for testing oral glucose tolerance. J. Jap. Diab. Soc. 11, 207-210 (1968) (in Japanese)

9. Hirata, Y., Nakamura, Y., Inuzuka, S., Katsuki, S.: Side effects during the standard glucose tolerance test and an improved new solution for the test. J. Jap. Diab. Soc. 11, 319-323 (1968)

10. Nakano, M., Ito, M., Sakon, J., Kondo, S. : Changes of blood sugar, insulin and non-esterified fatty acid after Trelan G50 and glucose loading. A comparative study. J. Jap. Diab. Soc. 15, 314-315 (1972)

11. Hagedorn, H.C., Jensen, B.N.: Zur Mikrobestirnmung des Blutzuckers mittels Ferricyanid. Biochem. Z. 135, $46-58(1923)$

12. Kanazawa, Y., Kuzuya, T., Ide, T., Kosaka, K.: Plasma insulin responses to glucose in femoral, hepatic and pancreatic veins in dogs. Amer. J. Physiol. 211, 442-448 (1966)

13. Kuzuya, N. and others: Report of the committee on the diagnostic criteria of the oral glucose tolerance test for diabetes mellitus. Recommendations on the evaluation of the oral glucose tolerance test for the diagnosis of diabetes mellitus. J. Jap. Diab. Soc. 13, 1-8 (1970) (in Japanese)

14. Ryan, W.G., Schwartz, T.B., Nibbe, A.F.: Serum Immunoreactive insulin levels during glucose tolerance and intensive islet stimulation. Diabetes 20, 404-409 (1971)

15. Katsura, E.: In Iwatsuru, R. (ed.), Theory and Practice of diet therapy (in Japanese), ed. 4., Nanzando, Tokyo P. 483 (1955)

16. Luft, R., Cerasi, E., Hamberger, C. A.: Studies on the pathogenesis of diabetes in acromegaly. Acta Endocr. (khb.) 56, 593-607 (1967)

17. Varsano-Aharon, N., Echemendia, E., Yalow, R.S., Berson, S.A.: Early insulin responses to glucose and 
to tolbutamide in maturity-onset diabetes. Metabolism 19, $409-417$ (1970)

18. Robertson, R.P., Porte, D. Jr.: The glucose receptor. A defective mechanism in diabetes mellitus distinct from the beta adrenergic receptor. J. clin. Invest. 52, $870-876(1973)$

19. Cerasi, E., Luft, R.: Further studies on healthy subjects with low and high insulin response to glucose infusion. Acta Endocr. (kbh.) 55, 305-329 (1967)

20. Siperstein, M.D., Norton, W., Unger, R.H., Madison, L. L.: Capillary basement membrane width in normal, diabetic and prediabetic patients. In Östman, J. (ed) Diabetes. Excerpta medica. International Congress Series Amsterdam 172, p. 572-581 (1969)

Dr. N. Kuzuya

The Institute of Adult Diseases,

Asahi Life Foundation

1-9-14 Nishishinjuku

Shinjuku-ku

Tokyo 160/Japan 